\title{
Analysis of suborbital flight operation using PESTLE
}

\begin{abstract}
Suborbital flight has progressively emerged as the potential future transport trend, whether for space tourism or other applications. While several design concepts have been explored thus far for the suborbital flight operation, one of the main lingering concerns is actually the destination of the flight. The altitude of $100 \mathrm{~km}$, which is the commonly chosen destination for suborbital flight, provides an imaginary separation between air and space. The quest for this separation sparks intense debates not only in the scientific communities but also in the political circles and legal fraternities. In conjunction with that, this research aims to analyse the suborbital flight operation by using the analytical tool of Political, Economic, Social, Technological, Legal and Environmental (PESTLE) in order to discuss its implications to a nation. From the analysis, among the advantages of suborbital flight operation include the shifting of role for space development from public agency to private enterprise, creation of new industry to generate revenue, public opportunity for access to space, new, low cost and reusable technology and material and also the enhancement of atmospheric studies. On the other hand, among established disadvantages of suborbital flight operation include the absence of international consensus on the boundary between air and space, intensive capital requirement to develop new technology and infrastructure, damage to third party liability, limited track record for flight safety, lack of regulation for new industry and also the air pollution resulting from the flight activity. All in all, it can be taken that there are many advantages that suborbital flight could bring to a nation but its subsequent disadvantages have to be carefully considered as well to ensure the sustainability of its operation and industry.
\end{abstract}

Keyword: Suborbital flight; PESTLE analysis; Space tourism; Space policy; Space laws 